\title{
Agent-Based Mobile Robots Navigation Framework $^{\star}$
}

\author{
Wojciech Turek, Robert Marcjan, and Krzysztof Cetnarowicz \\ Institute of Computer Science \\ AGH University of Science and Technology \\ al. Mickiewicza 30, Krakow, Poland \\ wojciech.turek@agh.edu.pl, marcjan@agh.edu.pl, cetnar@agh.edu.pl
}

\begin{abstract}
The problem of mobile robot navigation has received a noticeable attention over last few years. Several different approaches were presented, each having major limitations. In this paper a new, agentbased solution the problem of mobile robots navigation is proposed. It is based on a novel representation of the environment, that divides it into a number of distinct regions, and assigns autonomous software Space Agents to supervise it. Space Agents create a graph, that represents a high-level structure of the entire environment. The graph is used as a virtual space, that robot controlling agents work in. The most important features of the approach are: path planning for multiple robots based on most recent data available in the system, automated collision avoidance, simple localization of a "lost robot" and unrestricted scalability.
\end{abstract}

\section{Introduction}

The most crucial features, that a mobile robot must possess, are ability of reaching desired position and determining current location in the environment. Moreover, the robot must be able to adopt to changes that take place in the environment, and to cooperate with other robots of the same or different type to avoid collisions or deadlocks. A number of approaches to this type of problems have been described, but each has some significant drawbacks or limitations.

Single robot navigation problems are known in literature as the "Simultaneous Localization and Map Building" (SLAM 1 ) problem and the "Kidnapped Robot Problem". In the Kidnapped Robot Problem a robot "wakes up" in previously analysed environment; the task is to quickly estimate robot's position. In the SLAM problem a mobile robot is situated in an unknown environment, and is supposed to build a map of the environment basing only on own sensors readings. The map is simultaneously used for position estimation and path planning. Most popular approach to the SLAM problem 2] [5] is based on determining position of a number of distinguishable landmarks (artificial or natural) and assumption of Gaussian distributions of observed landmarks locations errors - Kalman filters [3] are used. These solutions allow single robot to determine own location and

\footnotetext{
* This work was partially supported by the grant MEiN Nr 3 T11C 03829.
} 
to maneuver in a limited and usually invariable environment. Dynamic environments problem has also received a noticeable attention - Janglov [7] proposed a neural networks approach, in [8] Hahnel et al. presented a method of building a dynamic map with probabilistic model of obstacle presence certainty.

These solutions show, that abilities of a single robot are strongly restricted - its computational power, memory or effectors abilities may be insufficient, it cannot observe dynamic changes of whole large environment. Most of these problems can be theoretically overcame by a group of cooperating robots - memory and computations can be distributed, a few robots can perform one task. However, some new problems with navigation appear, mostly concerning movement coordination, group path planning and collision avoidance.

Several approaches were proposed. In 6] Arras et al. present a solution, that uses a graph for navigation in EXPO exhibition centre. The graph must be created manually, therefore the solution is not universal. A reactive collision avoidance method, where robots detect collision situation and modify their velocity to avoid it, is described in [9]. The method does not solve the problems of localization and multirobots path planning.

Lack of universal solutions to the problems of multirobot path planning and localization in dynamic environments makes it difficult to perform research into advanced applications of multorobots systems. Tasks like automated guarding, cleaning, transportation or manufacturing require reliable solutions of robots mobility problems.

Most research into navigation problems situates an autonomous robot in an unknown, hostile environment, and makes the robot responsible for gathering and processing data, that describe the terrain. The Framework proposed in this paper is based on a different assumption: fragments of environment, as well as robots, are given some consciousness and autonomy - they are considered to be software agents [10]. This approach makes it possible to apply global and local path planning algorithms that operate on a distributed and up-to-date knowledge about the environment. The solution supports robots with ability of reaching desired position at desired time, using optimal path and without causing collisions with other robots or obstacles.

\section{Navigation System Architecture}

The physical space, that robots operate in, is divided into relatively small rooms. Each room is supervised by a single Space Agent (SA), that is responsible for gathering and processing information about assigned terrain. Every Space Agent is supposed to know its direct SA neighbours - these connections create SA graph, which represents a high-level structure of the entire environment. The SA graph is also considered a virtual space, that mobile Robot Agents work in.

A Robot Agent (RA) is closely associated with a single physical robot. It controls robot's movements, receives sensor readings, and can perform typespecific operations. At any moment every RA is registered in one SA, depending on its robots location. When it needs to relocate, it informs the SA about desired 
destination, and receives precise movement information. Simultaneously it sends to SA robot's sensors readings - this can be the only source of environment describing data. Due to autonomy and ability of communication, SAs are able to plan optimal, non-colliding paths for multiply robots, basing on most recent data available in the system.

This abstract architecture can be implemented in many different ways; two of them are described below.

\subsection{Virtual Agents Scenario}

More efficient and more demanding approach places software agents in a space, that is physically separated from the robots. The RAs and SAs are performing computations on computers in a network and control robots using radio connections. A communication infrastructure must cover entire environment, but processing can be faster and more complex when performed remotely. The architecture is presented in Figure 1.

There are many practical applications of this scenario, mostly concerning robots systems, that perform tasks like cleaning or guarding in the same place for a long time. An additional infrastructure is required, but also additional advantages appear. Devices like electric automated doors or elevators can be easily integrated with a corresponding SAs in order to increase robots functionalities.

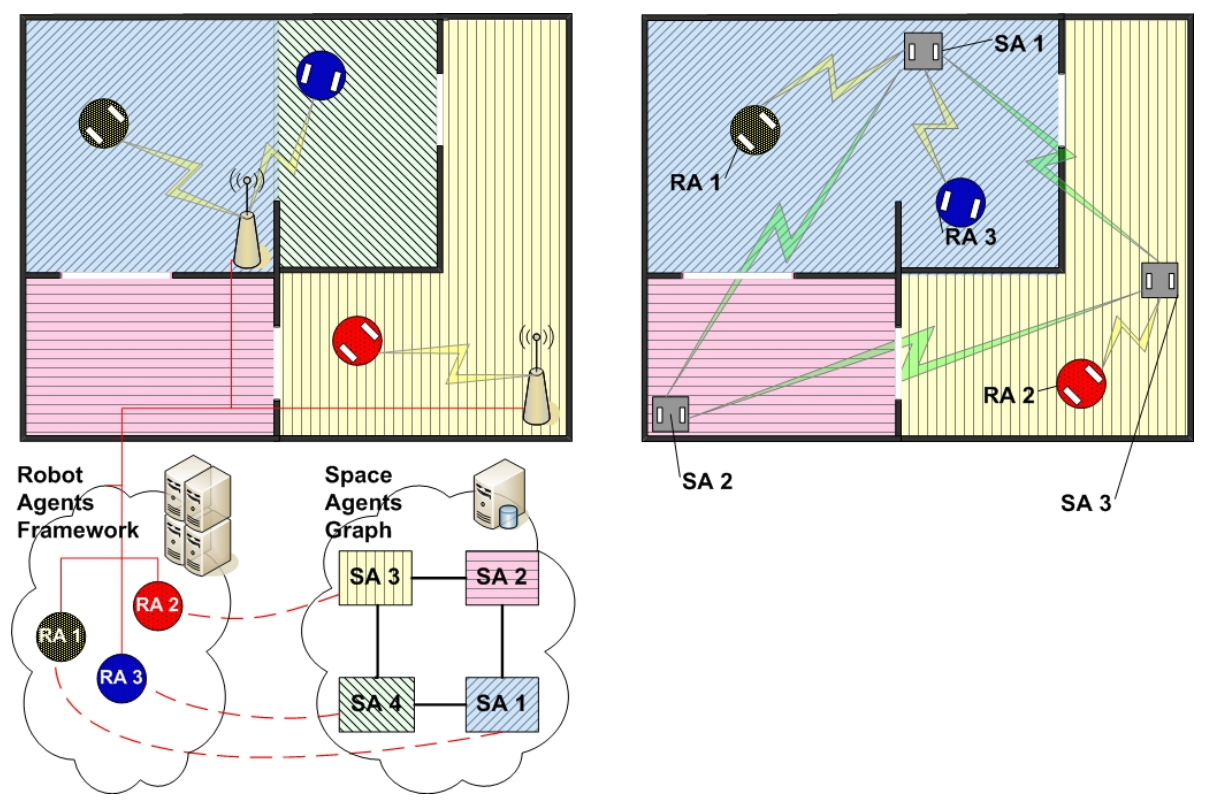

Fig. 1. Comparison of two implementations of the Navigation System: in an environment with communication infrastructure and external agent framework (on the left), and in an unknown environment with fully autonomous multirobot system (on the right) 
Moreover, the situation does not restrict a size of the environment - neither memory nor computational power, that is required to navigate in a huge space, are limited by robots capabilities.

\subsection{Hardware Agents Scenario}

Described Navigation Framework can also be successfully implemented, when no external infrastructure is available in the environment. There are some additional requirements though. A set of robots is required to build a physical graph of Space Agents. The number and location of those robots is determined by communication ranges and environment properties - the SA graph must be consistent, every pair of neighbour SAs should establish a direct connection. Unlike the previous scenario, the robots must offer a significant computational performance, because all data storing and processing must be performed them. An example is shown in Figure 1.

\section{Terrain Description Grid}

Each Space Agent is responsible for a given part of the environment. It must gather data that describes the terrain from robots, that are passing through, and support these robots with information, that will help them to move fast and safely. The problem, that must be solved by an SA is very similar to a typical SLAM, except multiple sources of data are available. The solution presented below is a novel approach that can be used with any set of sensors, that can support sufficient amount of information. The assumption is made, that every robot has almost the same sensor readings, when situated in almost the same location.

A Space Agent applies a kind of graph, called a description grid, on the assigned space, as shown in Figure 2 Each node of the grid has unique location, and is connected with up to eight neighbours. Distance between nodes should be greater than a diameter of the smallest robot's bounding circle, so that two robots situated in adjacent nodes would not collide.

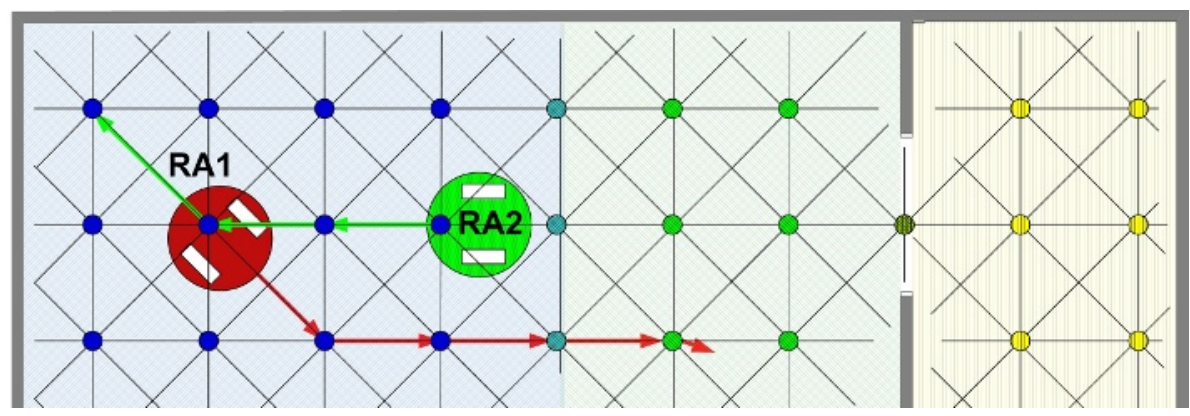

Fig. 2. Example of a terrain description grid, applied on a fragment of environment. Paths planned for two robots are not considered colliding. 
Nodes are used for storing a vector of values returned by sensors of a robot, when situated in the node. Depending on types of sensors used, size of the vector will vary. General rules concerning selection of sensors are:

- two description nodes, that have very similar sensor readings vector should never be adjacent

- perfect (complete) set of sensors ensures that there are no two nodes in the environment that have the same sensor readings vector

- size of the vector should not be large, therefore sensor readings should be possibly uncorrelated

- a method for determining which nodes are physically accessible is required (e.g. directional range measurement).

The paradox of the SLAM problem is, that a map is built from robot's sensors readings gathered at a particular location, which are simultaneously used for determining this location. This issue does not cause problems as long as the environment (or at least landmarks set) remains static. If dynamic changes can occur, a robot may report a sensor reading vector, that is significantly different than the previously stored one. The SA must then decide, whether the environment has changed rapidly or the RA estimated wrong location of the robot.

Each RA knows an approximate position of controlled robot. The approximation accuracy is defined by a certainty factor $C_{R} \in[0,1]$, which is reduced, as robot moves. When the robot reaches a node, its position is confirmed with SA, using stored sensor readings vectors, and robot's $C_{R}$ is increased. Each sensor readings vector value that is stored at a node is also connected with a certainty factor $C_{N} \in[0,1]$. If the readings vector is unknown: $C_{N}=0$, just after a robot reports current vector: $C_{N}=1$. The value is reduced as time passes, but once reported, never goes below 0.5 .

If a conflict between stored and sensed readings occurs, the value with a higher certainty factor is considered real. When a robot "is right", node's data is updated, when all robots's readings are significantly different from the stored ones, so that its position cannot be confirmed, $C_{R}$ is not increased. When $C_{R}$ reaches 0 , the robot is considered "lost" and kidnapped robot procedure is started.

There are two functions defined for each value in the sensor readings vector: inverted interpolation and propagation. The inverted interpolation function defines a method to calculate accurate robot's position basing on its sensor readings. If the description grid is dense enough, linear function can be used. The propagation function is used to update data in the nodes adjacent with the one the robot is located in - it is performed with reduced $C_{R}$ value. The function depends on sensor type, and usually can be defined only for selected directions of adjacency.

\section{Features of the Framework}

The framework described in this paper solves several problems concerning mobile robots navigation: 
Kidnapped robot problem. If a new RA is introduced to the system, it does not know its robot's location - the robot is considered "lost". Every SA in the system is then requested to find possible location, using robot's sensor readings vector. Provided, that the area, the robot is situated in, has already been covered by a SA's grid, location of the robot is estimated and robot's $C_{R}$ is set to a value just above 0 . If the location estimation is correct, the $C_{R}$ factor will rise, otherwise the robot will soon become "lost" again.

SLAM problem. Starting with one RA with a robot at $(0,0)$ and $C_{R}=1$, and one SA, the map will be incrementally built, without any additional methods needed. The only requirement is, that SA should be able to grow its grid, and to divide if necessary. New robots can be added to the system, with a "lost" status, as soon as a part of the environment is covered by SA's grid.

Multiply robots navigation with collision avoidance. Navigation algorithm is divided into two stages:

- Global route planning - current SA searches the SA graph to find every possible route to a desired location. Each route is then split and analysed the first, that meets given time requirements is selected.

- Local path finding - each SA in a particular route must plan robot's movements, so that it reaches the destination or moves to another SA. The SA receives initial location of the robot, time of reaching it, robot's size and maximum velocity, and desired destination coordinates. It is supposed to return guaranteed time of reaching the destination. If a SA has a complete information about assigned terrain, the local path finding can be considered a simple graph searching problem with constraints. Two types of constraints are possible: constant, when a node is physically inaccessible, and temporary, which occurs when a previously created path uses a particular node. Restricted nodes can be either omitted, or a robot may wait for node to become free.

After choosing the best route, a movement cycle begins:

- RA receives information about next location that should be reached by a robot

- robot moves, basing on previous location and some method of estimating covered distance (e.g. odometers readings, time and velocity calculations)

- sensor readings are sent to the $\mathrm{SA}$ in order to confirm current location and get next target coordinates

- SA verifies robots position and simultaneously updates information about the environment

Scalability. The environment description data is fully distributed, therefore scalability of the system is hardly restricted. If SA's memory or computational abilities turn out to be insufficient, it can always be divided, and each SA can work on a different computer in a network. The only restriction could be SAs 
graph searching algorithm, but in most typical applications the number of SAs will not be greater than a hundred, which is few.

Up-to-date knowledge availability. One of the most important advantages of the approach is, that all navigation is based on complete and most recent knowledge, that is available in the system. Therefore planned paths are always most optimal.

\section{Example of Implementation}

First implementation of the Navigation Framework was tested with RoBOSS Simulation System [11. A precise model of small soccer-playing robot is used; equipped with eight range finding sensors, an odometer and a compass. Nodes of the grid store readings from rangefinders; the data is used both for localization and for determining, which nodes are physically accessible. Manual methods for SA division are used; algorithms of path finding and lost robot localization are implemented.

The propagation function, started in a particular node, assigns an estimated values in nodes along rangefingers directions, with $C_{N}$ reduced by $10 \%$ each node. The inverted interpolation function uses readings from nine nodes $(3 \times 3$ square) to calculate eight lines, the robot should be situated on. Intersection points should be almost identical, indicating robot's accurate location; sum of distances between them is used for selecting best location of a "lost" robot.

Figure 3 shows a description grid and a SA graph generated for a simple maze. Three robots were able to reach randomly assigned locations without any problems, "lost" robot was also localized properly. Tests showed, that used set of sensors causes problems with accuracy, because even a very small error of compass readings causes large changes in values returned by rangefinders.
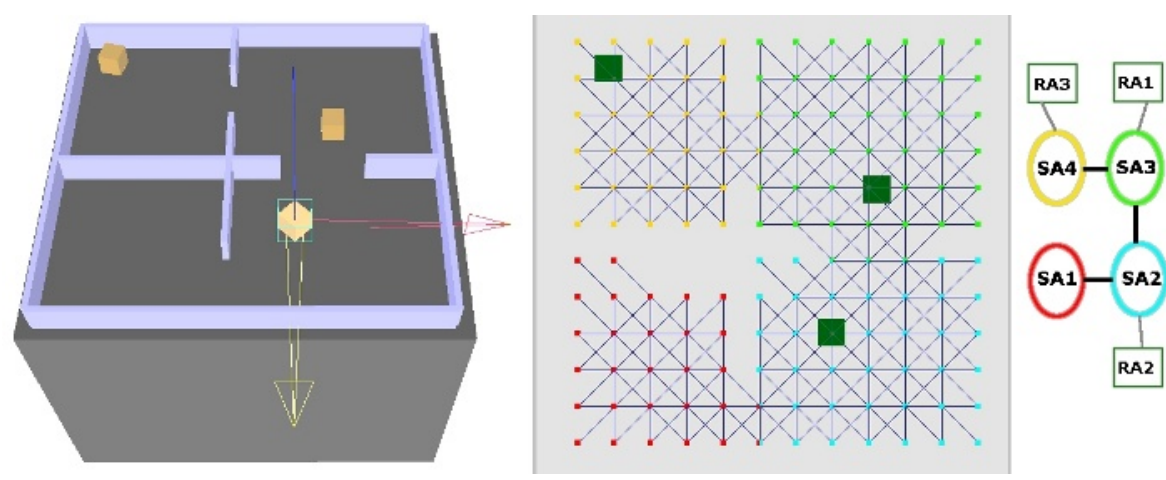

Fig. 3. Example of a simple environment with three FIRA MiroSot robots. A description grid, shown in the middle, is divided among four Space Agents. 


\section{Conclusions and Further Work}

The Navigation Framework proposed in this paper solves several problems concerning multirobot navigation. The idea of Space Agents graph distributes the data that describes the environment, solving scalability problems. Path planning algorithm based on up-to-date information, guarantees optimal and collision-free solutions.

The example showed, that use of range finding sensors only, may be insufficient in some cases, therefore further research into types of sensors is required. Tests with real robots are also planned to verify the approach.

\section{References}

1. Clark S., Dissanayake G., Newman P., Durrant-Whyte H.F.: A Solution to the Simultaneous Localization and Map Building (SLAM) Problem IEEE Transaction. on Robotics and Automation, vol. 17, no 3, 229-241, 2001

2. Guivant J., Nebot E. Optimization of the simultaneous localization and map building algorithm for real time implementation. IEEE Transaction on Robotics and Automation, vol. 17, no 3, 242-256, 2001

3. Glielmo L., Setola R., Vasca F.: An interlaced extended Kalman Filter. IEEE Transaction on Automatic Control, vol. 44, no 8, 1999.

4. Montemerlo M., Thrun S., Koller D., Wegbreit B.: FastSLAM: A Factored Solution to the Simultaneous Localization and Mapping Problem. In Proc. of the AAAI National Conference on Artificial Intelligence, Edmonton, Canada, 2002.

5. Se S., Lowe D., Little J.: Local and Global Localization for Mobile Robots using Visual Landmarks. In Proc. of the IEEE/RSJ International Conference on Intelligent Robots and Systems, Maui, Hawaii, USA, 2001.

6. Arras K. O., Philippsen R., de Battista M., Schilt M., Siegwart R.: A navigation framework for multiple mobile robots and its application at the Expo.02 exhibition. In Proc. of the IEEE/RSJ IROS, Lausanne, Switzerland, 2002.

7. Janglov D. Neural Networks in Mobile Robot Motion.: International Journal of Advanced Robotic Systems, vol. 1, no 1, 2004.

8. Hahnel D., Triebel R., Burgard W., Thrun S.: Map building with mobile robots in dynamic environments. In Proc. of the IEEE International Conference on Robotics and Automation, 1557-1563, 2003

9. Madhava Krishna K., Hexmoor H.: Reactive Collision Avoidance of Multiple Moving Agents by Cooperation and Conflict Propagation. In Proc. of the IEEE International Conference on Robotics and Automation, NewOrleans, 2004

10. Cetnarowicz K.: M-Agent Architecture Based Method of Development of Multiagent Systems In Proc. of 8th Joint EPS-APS International Conference on Physics Computing, Krakow, Poland, 591-596, 1996

11. Turek W., Czyrnek D., Marcjan R., Cetnarowicz K.: RoBOSS - an universal tool for robots modelling and simulation. In Proc. of Computer Methods and Systems, Krakow, Poland, 347-354, 2005 